\title{
Grassroots Business Intelligence as an Enabler of Change Management: A Case Study at a Large Global Manufacturing Firm
}

\author{
Makoto Nakayama $^{1 *}$, Öykü Isik ${ }^{2}$, Norma Sutcliffe ${ }^{1}$, and Sebastian Olbrich ${ }^{3}$ \\ ${ }^{1}$ College of Computing and Digital Media, DePaul University, Chicago, 60604, U.S.A. \\ ${ }^{2}$ IMD Business School, Lausanne, Switzerland \\ ${ }^{3}$ Capgemini, Mainzer Landstrasse 186, 60327 Frankfurt, Germany \\ MNakayama@cdm.depaul.edu, oyku.isik@imd.org, NSutcliffe@cdm.depaul.edu, \\ sebastian.olbrich@capgemini.com
}

\begin{abstract}
Business intelligence (BI) has attracted considerable attention in recent years. Despite the hype, BI frequently suffers from broad definitions, unrealistic expectations, and the incongruities of IT needs between headquarters and local business units. We report the findings of a case study at a large international manufacturing firm headquartered in the EU. The firm wants to transform itself from a conservative firm with traditional values to a more entrepreneurial and nimble organization with its supply chains driven by Industry 4.0 and the Internet of Things (IoT). The firm is going through its transformation, focusing on the BI system. Our observations suggest that firms consider BI to be the enabler of change management rather than simply technical tools that extend the traditional systems environment.

Keywords: Business Intelligence (BI), Industry 4.0, Big Data, The Internet of Things, Grassroots BI.
\end{abstract}

\section{Introduction}

Over the past decade, business intelligence (BI) has drawn considerable attention from both academic and practitioner communities [1]. In response to an ever-increasing amount of data to analyze and growing pressure to provide better and quicker responses to customers, many organizations have turned to BI applications as a means to improve organizational decision making.

Originally coined by the Gartner Group in the 1990s, BI typically refers to a variety of information technology (IT)-based tools and approaches for helping organizations make better use of the increasingly vast amounts of data accumulated from both internal and external sources. Thus, BI is typically defined as a system comprised of both technical and organizational

\footnotetext{
* Corresponding author

(C) 2020 Makoto Nakayama et al. This is an open access article licensed under the Creative Commons Attribution License (http://creativecommons.org/licenses/by/4.0).

Reference: M. Nakayama, Ö. Isik, N. Sutcliffe, and S. Olbrich, "Grassroots Business Intelligence as an Enabler of Change Management: A Case Study at a Large Global Manufacturing Firm," Complex Systems Informatics and Modeling Quarterly, CSIMQ, no. 23, pp. 1-11, 2020. Available: https://doi.org/10.7250/csimq.2020-23.01 
elements that presents information to its users for analysis and enables effective decision making and management support, with the overall purpose of increasing organizational performance [2].

Recently, the amount of data available has dramatically increased, leading to a new term being coined: big data. Big data has been described as a "critical new form of economic currency" and defined as "data sets that can no longer be easily managed or analyzed with traditional data management tools, methods, and infrastructures" [3]. Next to the mind-boggling increase in data, the rise of analytics and its use to guide decision making has also motivated organizations to leverage data for competitive advantage. Considering the close connection of BI with these new concepts, BI systems have also started to increasingly include and combine the use of predictive modeling, including (mostly unstructured) big data and sensor data from the so-called Internet of Things (IoT) within.

While BI has become a common term, the conceptualization and expectations from BI vary among organizations and individuals. It may encompass the mere description of functionality (e.g. reporting, consolidation) as well as the key elements of information management in large organizations (e.g. creating a corporate memory by means of data warehouses) to constitute a fundamental part of data science (e.g. by including analytical techniques). Hence, BI and analytics are usually referred to as one of the cornerstones of the digital transformation, which can be broadly defined as IT-enabled major business improvements [4]. As any transformation includes major change management efforts, we are particularly interested in the transformational power of BI in two ways: 1) What impact does digital transformation have on the roles of BI systems? 2) What transformational effects can BI systems support?

Seeking the answers to these research questions, we have the opportunity to observe an organizational transformation project at a large global manufacturing firm aspiring to become a leading Industry-4.0 firm [5]. Our research goal is to learn from this firm's journey towards accomplishing a transformational experience. In particular, this firm aims to change from a conservative firm with traditional values to a more entrepreneurial and nimble organization with its supply chains driven by Industry 4.0 and the IoT. We focus on the structuration effects [6] of digital transformation and BI systems at the grassroots level.

\section{Literature Review}

BI systems, especially for organizations operating in highly competitive environments, are critical. They are expected to contribute to decision making by providing users with timely access, effective analysis, and insightful presentation of data, collected from outside or generated within the organization, enabling them to make the right decisions [7], [8]. Thus, BI systems have so far been commonly identified as strategic enterprise-wide technological solutions holding quality information in well-designed data stores. Even though the majority of BI implementations are data-centric, focusing on reporting and advanced analytics, recent research confirms the existence and the need for process-centric BI applications [9]. Indeed, BI systems provide higher business value when used within management processes that impact operational processes, as well as within those operational processes themselves [10].

Research shows that the operational approach to BI increases the value gained from the BI system [11]. For instance, Bronzo et al. [12] show that business analytics, as a part of BI, have a positive effect on organizational performance when aligned with process orientation initiatives. However, operational or functional BI is sometimes seen as undesirable by the IT departments in organizations as it requires integrating BI into operational business processes. Thus, IT typically supports only the enterprise BI solutions such as data warehouse and dashboard use across units. As Arnott et al. [13] found after examining 86 decisions supported by the BI systems used at eight organizations, this leads to more than $80 \%$ of decisions being supported by corporate-wide BI systems, whereas fewer than $20 \%$ use departmental or unit-specific BI systems [13]. Moreover, current BI systems are predominantly geared towards non-strategic operational/tactical support, not necessarily fostering change and innovation at the grassroots 
level. This is also supported by other research that shows BI is traditionally used by IT-savvy leaders with a higher education [14].

For our case study, we briefly review the BI literature regarding terminology, commonly used theoretical/research perspectives, and an underrepresented perspective.

\subsection{BI Definitions}

Various definitions of BI have emerged in the academic and practitioner literature, typically using BI as an umbrella term [1], [15]. While some broadly define BI as a holistic and sophisticated approach to cross-organizational decision support, others approach it from a more technical point of view. The definition of BI can focus on (a) applications, infrastructure, tools, and best practices [15], (b) concepts and methods to improve business decisions [1], (c) the distribution of "the right information to the right people at the right time" [16, p. 22], (d) the purposeful use of data for decision making [17], and (e) the outcomes of information use in the business domain [18]. Recently, BI has also been defined as a system comprised of both technical and organizational elements that presents information for analysis to enable effective decision making and management support for users, with the overall purpose of increasing organizational performance [19]. These different approaches to defining BI also yield different perspectives on BI success or may lead to varying expectations from BI among managers and end-users.

\subsection{BI Perspectives}

Given the broad definitions of BI, we must consider a number of contextual factors of a sociotechnical nature that affect BI systems [14]. The perspectives put forward in BI review papers include structured vs. unstructured data sources [20], the BI category vs. research strategy [21], socio-technical evolution [22], knowledge management [23], and banking [24]. However, a common perspective is the data-centric approach because "getting data is the most challenging aspect of BI" [25, p. 96].

\subsection{BI Outcomes}

Organizations may implement BI to achieve a variety of organizational benefits; therefore, the expected outcomes, or how BI success is defined, may differ from organization to organization, depending on the benefits expected from the BI initiative [19]. Successful BI implementation provides "analytical tools to present complex internal and competitive information to planners and decision makers" [20, p. 178], self-service and performance measurement capabilities for individual managers [26], decision support, work integration, and improved customer service [11]. BI success also creates operational and strategic business values [27] as well as a competitive advantage and stakeholder satisfaction [28]. It is safe to generalize that BI success is related to the positive value an organization obtains from its BI investment [19]. While some organizations are interested in tangible and quantifiable outcomes and use explicit measures (e.g. return on investment (ROI) improvements or increased profitability), others are interested in observing intangible outcomes. These may include users' perception of BI as mission critical or amount of support from the stakeholders [19]. Specific BI expectations may even differ across organizational units; for instance, one unit may use BI to achieve better management of its supply chain, while another may adopt it to achieve better customer service.

\subsection{BI and Change Management}

Regardless of the expected outcomes of BI, an organization-wide implementation implies a significant change in the business processes of the organization. This is similar to the fact that an enterprise resource planning (ERP) system implementation modifies the way an organization 
operates [29]. It implies that user adoption is potentially the most critical factor. An organization can invest in the most advanced BI infrastructure or implement the latest BI tools, but all of that will not mean anything if users are not using the systems as they are supposed to be used [30]. Properly used BI systems lead to the reengineering of fundamental business processes, which is all about change. Change is a type of event involving organizational form, quality, or state over time [31]. We thus define change management for BI as an incremental or transformational process of modifying the organization's reporting structure progressively, operational quality, and culture by using data, information, and knowledge effectively. Business process change management is a strategy-driven organizational initiative that sets out to achieve a competitive advantage by balancing the forces between change and resistance [32]. Although user-oriented change management is regarded as a critical success factor for the implementation of BI systems [33], the maturity and adoption levels of process-centric BI remain low [34].

\subsection{Underrepresented Perspective}

The fundamental outcome of BI is to change the work carried out by individual workers and organizational units by using improved decisions based on timely and relevant information. The more widespread such changes are implemented, the more benefits the organization can reap from BI. Because business contexts are usually complex and constantly changing, especially with such evolutions as Industry 4.0, we propose that BI users adopt a cognitive approach that "declares knowledge or rules about how to manage activities based on the current state of the environment" [35, p. 181]. From this perspective, we posit that BI should be regarded as the enabler of change management rather than as a technical tool that extends the traditional systems environment. And yet, change management, especially at the grassroots level, is not deservedly addressed in the current literature.

In sum, BI is often understood in different ways. In particular, because it is heavily datacentric, scholars might have overlooked BI's potential as an agent of organizational change in the socio-technical context.

\section{Method}

This study is in the first phase of an empirical BI study at Strasbourg Industries (the firm name is disguised) headquartered in the European Union (EU). Strasbourg is a traditional manufacturing firm with a long history of global business success, especially in various types of automotive control systems. Recently, the IoT has become a "megatrend" for smart and connected automotive control systems [36]. Given the rise of the IoT, Strasbourg has set the inspiring vision of being a leading Industry-4.0 firm that not only incorporates the IoT into its automotive systems products but also leverages the IoT to create new business models.

The firm's computing vision is heavily headquarters-driven, with the emphasis on developing standardized global templates and then applying those globally with some degree of customization. While the firm has used multiple ERP systems and data warehousing techniques since the turn of the century, firm-wide BI implementation is still in its formative stage, with the global BI template implemented in its major business units and fine-tuned in North America, the firm's biggest market. This region is regarded as the forerunner of strategic BI initiatives, allowing the firm to establish successful BI templates for the other regions in the world market. We feel the traditional ERP-driven approach at various sites needs adjustment to meet the new vision that emphasizes a more innovative culture. In particular, the firm's leadership must consider BI's role beyond the extension of its ERP systems and data warehousing environment because business innovations often emerge at the skunkworks level [37].

Because the firm is in the early rollout phase of global BI implementation, it is beneficial that the research team not only observes the process but also shares findings to help managers. We thus use the action research approach that facilitates the resolution of practical problems while 
gaining scientific knowledge [38]. We are in the diagnostic stage of the action study in which we interviewed a key BI manager in the IT unit and then $10 \mathrm{BI}$ power users, who were chosen based on their business process knowledge and the expectation of leading other users [39] in North America, in order to assess the state of Strasbourg's BI implementation. The structures of the questions were as follows. For the BI manager, we asked for an overview of the BI strategy as well as of BI programs and projects, progress, and challenges. BI power users were asked about their managerial roles, business needs, BI expectations, communication with the IT unit, and transformational changes before, during, and after certain phase(s) of the BI implementation. The interviews were recorded, transcribed, and coded for analysis. We used descriptive and topic coding to evaluate interviewees' understanding of BI as a concept and tool, communication with IT staff, and current and future expectations. By examining the results (see Section 4), we then formulated two dimensions of change management to develop a data-driven management paradigm, as summarized in Figure 1 in Section 5.

\section{Tentative Results and Implications}

\subsection{Broad Expectations}

We asked the BI power users what BI meant and how they perceived BI functionality. Because the definition of BI is not as clear cut as it is presented in Section 2, the responses are, not surprisingly, varied. Some representative answers are as follows:

"I don't quite understand [what BI means]." [ERP Coordinator]

"To me, [BI is] a broad term. I know what it means. It's really the applications BusinessObjects, Crystal Reporting, and creating dashboards - that basically allow for cockpits, for the organizations to use, and have the information together from the end-user all the way up to the top-level management." [Manufacturing Systems Engineer]

"[To me, BI is what the IT staff in the headquarters] designed canned reports that everyone uses." [Analyst]

“[To me, BI] is user-driven. It has changed how users interface with data." [ERP Specialist]

"When I say BI, I think of something that has source data, probably from ERP, feeding the data to cubes to do analytics, especially predictive analytics by using historical data." [Sales Forecasting Staff]

End-users generally perceive BI as a data-driven initiative that extends the scope of ERP systems. However, the expectation of BI goes beyond more standardized, visual reports and explicitly includes the transformation of business and organizational culture. Selected statements are below:

"[BI] is really for me that you can slice and dice the way you want." [Controlling Manager]

"There is no IT solution that can fix what I'm doing. It's just not there. I don't think there will ever be a complete IT solution for forecasting; there are too many pieces at play." [Sales Forecasting Staff]

"[The future of BI is geared towards] developing new business models to provide new revenue streams for Strasbourg [along with the IoT]. ... Yes, there is a big push to change the mindset of employees." [BI IT Manager] 
Some end-users anticipate that BI will bring new insights and ideas that go beyond current business practices.

\subsection{Lateral Communication of the BI Vision}

Communication of the vision is critical in any enterprise-wide system implementation between management and end-users. Because BI is contextual to business processes, BI end-users invariably indicate that the communication between business units and IT has been challenging. This raises the question of whether an internal IT department is the right partner for supporting BI.

"Communication is good as long as we are in the same building [with the IT department]." [Sales Forecasting Manager]

"IT groups can tell you the umbrella concepts. But IT is not supposed to understand (the details of) different businesses underneath. They regard themselves as support providers. Being proactive solution providers is not in their scope." [Finance Manager]

"The main challenge has been to have IT staff understand [our] business context. In addition, there are communication barriers when I talk to IT staff [overseas].” [Logistics Analyst]

"To be honest, directly with the IT people, I don't communicate unless we have them on site." [Manufacturing Systems Engineer]

"I use Strasbourg Connect [a collaboration/knowledge-sharing website]. I posted questions four times, but got only one short reply. Strasbourg Connect has not been helpful so far." [Logistics Analyst]

Despite these communication barriers, end-users do not expect their IT colleagues to fully comprehend the details of their operational needs, as seen in the second quote above from the Finance Manager. Recalling the last two quotes from the ERP Specialist and Sales Forecasting Manager in the previous section, what end-users implicitly suggested is what we call grassroots $B I$, in which BI end-users drive tool selections and implementation to pioneer ecological grassroots innovation [40]. The term "BI sandbox" [41] refers to the testing environment for BI end-users. Grassroots BI, on the contrary, goes beyond analytical solutions to influence work culture and foster innovation.

\subsection{BI as an Enabler of Change Management}

Because the firm has enjoyed long-term business success, its organizational structure and culture appear resistant to changes that may disrupt the status quo. Some users foresee BI as a potential catalyst for organizational change through data-driven innovation.

"The biggest challenge (for BI) is breaking barriers. Our organizational culture is still not into simplicity." [Unit Manager]

"We are nowhere near what technologies can do as far as I can see, for instance, at Gartner conferences." [Sales Forecasting]

“There are 'silos' between HR, finance, manufacturing, and operations within [our] division. Great BI applications should help overcome these 'silos." [Data Analyst] 
The use of analytics is evolving as part of the ongoing transformation. From a technological perspective, changes have occurred with the ever-growing amount of data. However, procedures are needed to analyze and integrate data into the organizational context. Because data analytics is one of the cornerstones of the digital transformation, we are slightly surprised that the organizational transformation towards working with data is still neglected, according to our interview data.

\section{Change Management Towards a Data-Driven Paradigm}

We borrowed open and axial coding elements from the grounded theory approach [42], [43] to investigate the changes in the organization in more detail. By coding our sample based on the interview data, we observed two dimensions of change management: a) the need to shift the management culture towards a data-driven work environment (e.g. "there is a big push to change the mindset of employees"; "Our organizational culture is still not into simplicity.") and b) the need to facilitate the changes needed to update the systems landscape (e.g. "The main challenge has been to have IT staff understand [our] business context"; "Great BI applications should help overcome these "silos."'). Following that logic, we derived a matrix of change management towards a data-driven management paradigm (Figure 1). In later research, we will use this matrix to guide our investigations.

Traditionally, BI is used to support decision making in organizations; some implementations even automate decision making (see the upper left quadrant in Figure 1). Mostly, Inmon's [44] criteria for data warehousing architecture serve as the design principle. Hence, the classic decision support role of BI is based on a dispositive data logic deeply rooted in financial information and structured standardized reporting. Previous research indicates that how closely BI reflects the goals of the organization in decision outcomes as well as the structure of the decisions for which the BI is used impacts BI outcomes [2], [45], [46]. The classic decisionsupport role of BI typically implies structured decisions presented in standard reporting tools or dashboards, mostly consumed by middle and upper management.

Expanding the scope of BI towards prediction indicates not only the changing nature of methods of analysis but also the changing sources of data. While BI traditionally feeds from internal data sources, during recent years, the field of BI has been adapting to deal with "bigger" data sources. With the entrance of "big data" into the business world, there is a growing demand to "know more" about the external environment and extract information by combining seemingly unrelated data sources, mostly collected from third parties. This asks for technologies and processes that are able to process greater amounts of data in different formats and structures (usually summarized by the three V's of volume, variety, and velocity). In addition to the changing design assumptions and system requirements, the obvious challenge is to integrate data sources into the existing technology stack in order to derive valuable insights for the organization. In some cases, the technologies and methodologies used should also be able to cope with real-time or near real-time analysis of streaming data. As the lower left quadrant of Figure 1 summarizes, only the sound integration of existing and emerging data sources can lead to viable predications, and this comes with a high level of change in the way BI is used.

The second change dimension (leading to the upper right quadrant of the matrix in Figure 1) addresses the raised expectations of BI users, and encompasses the steering function of BI. For modern users, who are accustomed to easily consumable IS services, long procedures of data gathering and siloed analysis are simply no longer acceptable. The changing expectations of endusers from their decision-making environments are also visible in the operational BI movement [9]. Operational BI centers around integrating BI into an organization's business processes and making it more flexible and dynamic. Operational BI addresses the need to "synchronize the efforts of decision-makers at the strategic, tactical, and operational levels to reach a common set of business goals and objectives" [9, p. 9]. Considering the trend and business expectation towards agility and faster decision making, BI could be used to automate structured and 
repetitive business processes, thus leaving more time and autonomy for the users to focus on unstructured cases. It will also enable the users to start forecasting the future instead of reporting on the past. It is important to note that this calls for a high level of process maturity in the organization. For instance, many well-known online retailers routinely suggest the next best deals. Why not allow BI systems to provide preliminary analysis results based on up-to-date data that could then be incorporated into an established, more traditional organization with sufficient IT resources? Such expectations raise the level of process maturity with fully automated decision making beyond quality reports, churn and fraud analytics, and so forth.

\begin{tabular}{|c|c|c|}
\hline & \multicolumn{2}{|c|}{ Process Change Towards Data-Driven Paradigm } \\
\hline 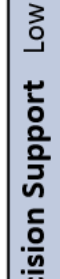 & $\begin{array}{l}\text { Classic Decision Support role of BI } \\
-\quad \text { BI as support function; e.g. Management } \\
\text { / Executive Information System } \\
-\quad \text { KPI mostly routed in financials } \\
-\quad \text { BI for standard reporting, dash-boarding } \\
-\quad \text { Mostly supported by dispositive data } \\
\quad \text { storage - e.g. data warehousing }\end{array}$ & $\begin{array}{l}\text { Steering function of BI } \\
-\quad \text { Decisions are based in key figures about } \\
\text { market and internal capabilities } \\
-\quad \text { Automated and structured decision process } \\
\text { (e.g. production / trading) } \\
-\quad \text { Integration of organizational functions } \\
\text { towards collaborative decision making }\end{array}$ \\
\hline 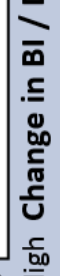 & $\begin{array}{l}\text { Expanding Scope towards prediction } \\
\text { - } \quad \text { (Big) Data is also collected outside the } \\
\text { organization } \\
-\quad \text { Advanced algorithms and Analytics are } \\
\text { applied to create insights } \\
-\quad \text { Challenges are modeling, integration and } \\
\text { reliability of data }\end{array}$ & $\begin{array}{l}\text { Data Driven Systems / Management } \\
\text { - Change in management culture towards a } \\
\text { new paradigm } \\
\text { - Organizational structures and procedures } \\
\text { lose meaning } \\
\text { - (Modeled) Context and boundary conditions } \\
\text { are key }\end{array}$ \\
\hline
\end{tabular}

Figure 1. Change Management Matrix towards a Data-Driven Paradigm

Whether an organization is driven by raised expectations or novel analytical capabilities (Strasbourg tends to be a case of the latter), the result will ultimately be a data-driven organization. Such data-driven modes of working depend on deriving sound figures rather than formal (hierarchical) structures and standardized procedures. By contrast, such legacy structures are often a major obstacle to a data-driven paradigm unleashing its full potential. In that light, the need articulated by our participants for holistic change management is understandable, as described in the lower right quadrant of the matrix in Figure 1. At the end of the day, technical perfection does not guarantee BI success; the most critical factor is business users embracing the BI system [30]. One possible direction of change for Strasbourg is to integrate Holacracy, a selforganization system in which (1) the organizational structure is team-based, (2) teams design and govern themselves, and (3) leadership is contextual [47]. The real business value of BI is seen when it is used within management as well as operational processes that directly drive revenue or reduce costs [9].

As organizations move towards expanding their BI use to handle a wider variety of decisions with managerial or operational nature and within various levels in the organization, it is important to pay attention to the variety of capabilities that BI provides to support decision making and how it is seen by top management. As the organization starts shifting from backward-looking questions such as "what happened?" towards forward-looking, "what will happen?". The role of BI will also shift towards a more steering function that leads the organization towards a data-driven paradigm. Certainly, future research should further investigate how optimally such organizational change is planned and facilitated. 


\section{Conclusions and Outlook}

This study reports findings of a case study regarding how a large established manufacturing firm is going through its transformation with BI. This company aims to transform and position itself as a market leader with Industry 4.0 and the IoT. We started the study with semi-structured explorative interviews that initially aimed to shed light on the alignment of global BI initiatives.

Given that the interviewees are from one organization, the findings may be biased to the organizational nature of Strasbourg Industries. Hence, the obvious next step is to apply methodological rigor and work with a hypothesis to solidify our results. Yet, sufficient data were obtained to articulate preliminary findings and postulate a research framework (Figure 1) for further investigation. During the first phase of our study, we found an interesting and probably under-investigated topic of change management for the digital transformation: changing the current mode of working from operations/procedures towards a data-driven paradigm with what we call grassroots BI. To foster grassroots innovations at the end-user level, we posit BI as the enabler of change management rather than technical tools that extend the traditional systems environment. Taking this fresh look at BI and analytics allows for a novel framing of digital transformation towards a data-driven paradigm. Even though bringing a new way of doing business has been suggested as a benefit of using BI [48], how BI can be a facilitator of change management has not been addressed in the literature to the best of our knowledge.

Our results indicate that such change management for the digital transformation will be a significant challenge, particularly for large, established organizations. In particular, leading tech companies that work with advanced analytics leveraging the IoT and Industry 4.0 will be the cornerstones of the digital transformation. We are grateful to have the opportunity to witness the organizational evolutions of a market leader. To validate these findings, we plan to include more organizations in the study to identify and isolate the organizational context as a moderating variable. Further, an organizational unit needs to align its information system use with the prevalent decision-making styles to enhance its effectiveness [49]. Future studies should examine how a unit's prevalent decision-making styles and leadership categories (directive, interactive, and non-directive) [49] moderate the effectiveness of change management towards a data-driven paradigm.

\section{References}

[1] H. Chen, R. H. Chiang, and V. C. Storey, "Business intelligence research," MIS Quarterly, vol. 34, no. 1, pp. 201-203, 2010.

[2] O. Isik, M. C. Jones, and A. Sidorova, "Business intelligence (BI) success and the role of BI capabilities," Intelligent systems in accounting, finance and management, vol. 18, no. 4, pp. 161-176, 2011. Available: https://doi.org/10.1002/isaf.329

[3] S. Rogers, "Big Data is Scaling BI and Analytics - Data growth is about to accelerate exponentially - Get ready," Information Management-Brookfield, vol. 21, no. 5, p. 14, 2011.

[4] M. Fitzgerald, N. Kruschwitz, D. Bonnet, and M. Welch, "Embracing digital technology: A new strategic imperative," MIT Sloan Management Review, vol. 55, no. 2, pp. 1-12, 2014.

[5] J. Lee, B. Bagheri, and H.-A. Kao, "A cyber-physical systems architecture for industry 4.0-based manufacturing systems," Manufacturing Letters, vol. 3, pp. 18-23, $2015 . \quad$ Available: https://doi.org/10.1016/j.mfglet.2014.12.001

[6] A. Giddens, The constitution of society: Outline of the theory of structuration. Univ of California Press, 1984.

[7] A. Popovič, P. S. Coelho, and J. Jaklič, "The impact of business intelligence system maturity on information quality," Information Research, vol. 14, no. 4, December 21, 2009. Available: https://ssrn.com/abstract=1625573.

[8] T. Peters, Ö. Işık, O. Tona, and A. Popovič, "How system quality influences mobile BI use: The mediating role of engagement," International Journal of Information Management, vol. 36, no. 5, pp. 773-783, 2016. Available: https://doi.org/10.1016/j.ijinfomgt.2016.05.003 
[9] O. Marjanovic, "The next stage of operational business intelligence: Creating new challenges for business process management," 40th Annual Hawaii International Conference on System Sciences, (HICSS 2007), IEEE, pp. 215c-215c. Available: https://doi.org/10.1109/HICSS.2007.551

[10] S. Williams and N. Williams, "The business value of business intelligence," Business Intelligence Journal, vol. 8, pp. 30-39, 2003.

[11] T. Grublješič and J. Jaklič, "Three Dimensions of Business Intelligence Systems Use Behavior," International Journal of Enterprise Information Systems, vol. 10, no. 3, pp. 62-76, 2014. Available: https://doi.org/10.4018/ijeis.2014070105

[12] M. Bronzo, P. T. V. de Resende, M. P. V. de Oliveira, K. P. McCormack, P. R. de Sousa, and R. L. Ferreira, "Improving performance aligning business analytics with process orientation," International Journal of information management, vol. 33, no. 2, pp. 300-307, $2013 . \quad$ Available: https://doi.org/10.1016/j.ijinfomgt.2012.11.011

[13] D. Arnott, F. Lizama, and Y. Song, "Patterns of business intelligence systems use in organizations," Decision Support Systems, 2017. Available: https://doi.org/10.1016/j.dss.2017.03.005

[14] S. Olbrich, J. Poppelbuß, and B. Niehaves, "Critical contextual success factors for business intelligence: A Delphi study on their relevance, variability, and controllability," 2012 45th Hawaii International Conference on System Science (HICSS), Hawaii, USA, IEEE, 2012, pp. 4148-4157. Available: https://doi.org/10.1109/HICSS.2012.187

[15] Gartner. "Business Intelligence (BI)." Gartner. Available: http://www.gartner.com/it-glossary/businessintelligence-bi/ (accessed November 13, 2016).

[16] R. M. Bogza and D. Zaharie, "Business intelligence as a competitive differentiator," 2008 IEEE International Conference on Automation, Quality and Testing, Robotics, Cluj-Napoca, Romania, 22-25 May 2008, vol. 1, pp. 146-151, 2008. Available: https://doi.org/10.1109/AQTR.2008.4588724

[17] L. De Leon, P. D. Rafferty, and R. Herschel, "Replacing the annual budget with business intelligence driverbased forecasts," Intelligent Information Management, vol. 4, no. 1, p. 6, 2012. Available: https://doi.org/10.4236/iim.2012.41002

[18] W. Chung, H. Chen, and J. F. Nunamaker Jr, "A visual framework for knowledge discovery on the Web: An empirical study of business intelligence exploration," Journal of Management Information Systems, vol. 21, no. 4, pp. 57-84, 2005. Available: https://doi.org/10.1080/07421222.2005.11045821

[19] Ö. Iş1k, M. C. Jones, and A. Sidorova, "Business intelligence success: The roles of BI capabilities and decision environments," Information \& Management, vol. 50, no. 1, pp. 13-23, 2013. Available: https://doi.org/10.1016/j.im.2012.12.001

[20] S. Negash, "Business Intelligence," Communications of the Association for Information Systems, vol. 13, no. 1, pp. 177-195, 2004. Available: https://doi.org/10.17705/1CAIS.01315

[21] Z. Jourdan, R. K. Rainer, and T. E. Marshall, "Business Intelligence: An Analysis of the Literature," Information Systems Management, vol. 25, no. 2, pp. 121-131, 2008 . Available: https://doi.org/10.1080/10580530801941512

[22] H. Chen, R. H. Chiang, and V. C. Storey, "Business Intelligence and Analytics: From Big Data to Big Impact," MIS Quarterly, vol. 36, no. 4, pp. 1165-1188, 2012. Available: https://doi.org/10.2307/41703503

[23] G. Muhammad, J. Ibrahim, Z. Bhatti, and A. Waqas, "Business Intelligence as a Knowledge Management Tool in Providing Financial Consultancy Services," American Journal of Information Systems, vol. 2, no. 2, pp. 2632, 2014. Available: http://pubs.sciepub.com/ajis/2/2/1.

[24] S. Moro, P. Cortez, and P. Rita, "Business intelligence in banking: A literature analysis from 2002 to 2013 using text mining and latent Dirichlet allocation," Expert Systems with Applications, vol. 42, no. 3, pp. 13141324, 2015. Available: https://doi.org/10.1016/j.eswa.2014.09.024

[25] H. J. Watson and B. H. Wixom, "The current state of business intelligence," Computer, vol. 40, no. 9, pp. 9699, 2007. Available: https://doi.org/10.1109/MC.2007.331

[26] M. D. Peters, B. Wieder, S. G. Sutton, and J. Wakefield, "Business intelligence systems use in performance measurement capabilities: Implications for enhanced competitive advantage," International Journal of Accounting Information Systems, vol. 21, pp. 1-17, $2016 . \quad$ Available: https://doi.org/10.1016/j.accinf.2016.03.001

[27] L. Fink, N. Yogev, and A. Even, "Business Intelligence and Organizational Learning: An Empirical Investigation of Value Creation Processes," Information \& Management, 2016. Available: https://doi.org/10.1016/j.im.2016.03.009 
[28] S. Rouhani, A. Ashrafi, A. Zare, S. Afshari, Z. Irani, and Z. Irani, "The impact model of business intelligence on decision support and organizational benefits," Journal of Enterprise Information Management, vol. 29, no. 1, pp. 19-50, 2016. Available: https://doi.org/10.1108/JEIM-12-2014-0126

[29] D. Aloini, R. Dulmin, and V. Mininno, "Risk management in ERP project introduction: Review of the literature," Information \& Management, vol. 44, no. 6, pp. 547-567, 2007. Available: https://doi.org/10.1016/j.im.2007.05.004

[30] W. Eckerson, "Transforming Finance: How CFOs Use Business Intelligence to Turn Finance from Record Keepers to Strategic Advisors: TDWI 2010 Q1 Best Practices Report,” TDWI, 2010. Accessed: January 18, 2018. Available: https://tdwi.org/research/2010/01/bpr-1q-transforming-finance-to-strategic-advisors.aspx

[31] A. H. Van de Ven and M. S. Poole, "Explaining development and change in organizations," Academy of Management Review, vol. 20, no. 3, pp. 510-540, 1995. Available: https://doi.org/10.5465/amr.1995.9508080329

[32] W. J. Kettinger and V. Grover, "Special section: toward a theory of business process change management," Journal of Management Information Systems, vol. 12, no. 1, pp. 9-30, 1995. Available: https://doi.org/10.1080/07421222.1995.11518068

[33] W. Yeoh and A. Popovič, "Extending the understanding of critical success factors for implementing business intelligence systems," Journal of the Association for Information Science and Technology, vol. 67, no. 1, pp. 134-147, 2016. Available: https://doi.org/10.1002/asi.23366

[34] T. Bucher, A. Gericke, and S. Sigg, "Process- centric business intelligence," Business Process Management Journal, vol. 15, no. 3, pp. 408-429, 2009. Available: https://doi.org/10.1108/14637150910960648

[35] M. Wang and H. Wang, "From process logic to business logic - A cognitive approach to business process management," Information \& Management, vol. 43, no. 2, pp. 179-193, 2006. Available: https://doi.org/10.1016/j.im.2005.06.001

[36] Automotive Megatrends. "Interview: Elliot Garbus, President, Internet of Things Solutions Group and General Manager, Automotive Solutions, Intel Corporation." Available: https://automotivemegatrends.com/interviewelliot-garbus-president-internet-things-solutions-group-general-manager-automotive-solutions-intelcorporation/ (accessed April 1, 2017).

[37] E. M. Rogers, Diffusion of innovations, fourth ed. New York: Free Press, 1995.

[38] R. Baskerville and M. D. Myers, "Special issue on action research in information systems: Making IS research relevant to practice: Foreword," MIS Quarterly, vol. 28, no. 3, pp. 329-335, 2004. Available: https://doi.org/10.2307/25148642

[39] O. Volkoff, M. B. Elmes, and D. M. Strong, "Enterprise systems, knowledge transfer and power users," The Journal of Strategic Information Systems, vol. 13, no. 4, pp. 279-304, 2004. Available: https://doi.org/10.1016/j.jsis.2004.11.004

[40] P. D. Jennings and P. A. Zandbergen, "Ecologically sustainable organizations: An institutional approach," Academy of Management Review, vol. 20, no. 4, pp. 1015-1052, 1995. Available: https://doi.org/10.5465/amr.1995.9512280034

[41] R. L. Sallam, J. Richardson, J. Hagerty, B. Hostmann, and Gartner, "Magic Quadrant for Business Intelligence Platforms," 2011.

[42] B. G. Glaser and A. L. Strauss, The discovery of grounded theory: Strategies for qualitative research, seventh ed. New Jersey: Transaction publishers, 2009.

[43] M. D. Myers, Qualitative research in business and management, second ed. London: Sage Publication, 2013.

[44] W. Inmon, Building the Data Warehouse. New York: John Wiley \& Sons, Inc., 1992.

[45] D. Arnott, "Decision support systems evolution: framework, case study and research agenda," European Journal of Information Systems, vol. 13, no. 4, pp. 247-259, 2004. Available: https://doi.org/10.1057/palgrave.ejis.3000509

[46] T. D. Clark, M. C. Jones, and C. P. Armstrong, "The dynamic structure of management support systems: theory development, research focus, and direction," MIS Quarterly, vol. 31, no. 3, pp. 579-615, 2007. Available: https://doi.org/10.2307/25148808

[47] E. Bernstein, J. Bunch, N. Canner, and M. Lee, "THE BIG IDEA Beyond the Holacracy HYPE," Harvard Business Review, vol. 94, no. 7-8, pp. 38-49, 2016.

[48] C. M. Olszak, "Toward better understanding and use of Business Intelligence in organizations," Information Systems Management, vol. 33, no. 2, pp. 105-123, $2016 . \quad$ Available: https://doi.org/10.1080/10580530.2016.1155946

[49] M. Nakayama and N. G. Sutcliffe, "The role of leadership decision styles on the use and effectiveness of Information Systems," International Journal of Information Systems and Change Management, vol. 3, no. 1, pp. 3-15, 2008. Available: https://doi.org/10.1504/IJISCM.2008.019286 